\title{
Inhibitory Effects of Coumarins from the Stem Barks of Fraxinus rhynchophylla on Adipocyte Differentiation in 3T3-L1 Cells
}

\author{
Eunjin Shin, Kyeong-Mi Chor, Hwan-Soo Yoo, Chong-Kil Lee, Bang Yeon Hwang, \\ and Mi Kyeong LEE* \\ College of Pharmacy, Chungbuk National University; Cheongju 361-763, Korea. \\ Received January 7, 2010; accepted May 21, 2010; published online June 8, 2010
}

In the course of screening anti-adipogenic activity of natural products employing the preadipocyte cell line, 3T3-L1 as an in vitro assay system, the EtOAc fraction of the stem barks of Fraxinus rhynchophylla DENCE (Oleaceae) showed significant inhibitory activity on adipocyte differentiation as assessed by measuring fat accumulation using Oil Red $O$ staining. Activity-guided fractionation led to the isolation of six coumarins such as esculetin (1), scopoletin (2), fraxetin (3), fraxidin (4) esculin (5) and fraxin (6). Among the six coumarins isolated, esculetin (1) showed the most potent inhibitory activity on adipocyte differentiation, followed by fraxetin (3). Further studies with interval treatment demonstrated that esculetin (1) exerted inhibitory activity on adipocyte differentiation when treated within $2 \mathrm{~d}$ (days $0-2$ ) after differentiation induction. We further investigated the effect of esculetin (1) on peroxisome proliferator activated receptor $\gamma(\operatorname{PPAR} \gamma)$, one of the early adipogenic transcription factors. Esculetin (1) significantly blocked the induction of PPAR $\gamma$ protein expression and inhibited adipocyte differentiation induced by troglitazone, a PPAR $\gamma$ agonist. Taken together, these results suggest that esculetin (1), an active compound from $F$. rhynchophylla, inhibited early stage of adipogenic differentiation, in part, via inhibition of PPAR $\gamma$-dependent pathway.

Key words Fraxinus rhynchophylla; adipocyte differentiation; esculetin; 3T3-L1; coumarin

Obesity arises from an imbalance in energy intake and energy expenditure, which leads to increased total fat mass. Obesity is no longer considered only a cosmetic problem but associated with several pathological disorders, including diabetes, hypertension, atherosclerosis and cancer. Thus, obesity is a highly prevalent health risk in industrialized countries. $^{1-3)}$

Adipose tissue is an important metabolic organ that is crucial for insulin sensitivity and energy homeostasis. Adipocytes also secrete molecules known as adipokines, which regulate physiologic processes such as glucose metabolism, appetite and inflammatory responses. In obesity, adipocytes undergo abnormal growth characterized by increased numbers of fat cells storing their lipids, which causes dysfunction of glucose and lipid metabolism and consequent pathological disorders. Pathological adipocyte growth can be achieved by the processes of mitogenesis and differentiation, regulated by diverse factors. Therefore, inhibition of mitogenesis of preadipocytes and their differentiation to adipocytes would be beneficial to prevent the initiation and progression of obesity. ${ }^{4-6)}$

Natural products have been used in the treatment of various diseases for a long time, especially in Asian countries. Related to adipogenic differentiation, various natural products such as epigallocatechin gallate, quercetin and resveratrol have been suggested to inhibit adipocyte differentiation with different mechanisms. ${ }^{7-11)}$ Therefore there is a growing interest in searching for adipogenic differentiation-inhibitory compounds from natural products with few adverse effects.

Fraxinus rhynchophylla DENCE (Oleaceae) is a traditional medicinal plant widely used in Asia. The stem barks of $F$. rhynchophylla have been used as antibacterial, analgesic and anti-inflammatory agents. In addition, diuretic, anticoagulant and antiallergic effects have been reported by pharmacological studies. ${ }^{12,13)}$ Related to its chemical constituents, coumarins are the characteristic constituents of Fraxinus species and responsible for the diverse activity such as antivirus and anti-inflammatory activity. ${ }^{14,15)}$ Besides coumarins, other constituents such as lignans, secoiridoid glycosides and other phenolic compounds were also reported. ${ }^{16-19)}$ In the course of screening adipocyte differentiation-inhibitory activity of natural products employing the mouse preadipocyte cell line, 3T3-L1 as an in vitro assay system, the EtOAc fraction of $F$. rhynchophylla showed significant inhibitory activity on adipogenesis. Thus, in the present study, we attempted to isolate the active constituents from $F$. rhynchophylla and evaluate their inhibitory activity.

\section{MATERIALS AND METHODS}

Plant Materials The stem barks of F. rhynchophylla were purchased from the local herbal market, Chungbuk, Korea in May 2009. They were identified by the herbarium of College of Pharmacy at Chungbuk National University, where a voucher specimen was deposited (CBNU200905FR).

Extraction and Isolation The stem barks of $F$. rhynchophylla $(6.0 \mathrm{~kg})$ were extracted 3 times with $80 \% \mathrm{MeOH}$, which yielded the methanolic extract $(595 \mathrm{~g})$. The methanolic extract was then suspended in $\mathrm{H}_{2} \mathrm{O}$ and partitioned successively with $n$-hexane, $\mathrm{CHCl}_{3}$, EtOAc and $n$-BuOH. The EtOAc fraction $(50 \mathrm{~g})$, which showed most potent activity, was subjected to further chromatographic separation. Compound 1 ( $3200 \mathrm{mg}$ ) was obtained from EtOAc fraction by recrystallization using $\mathrm{MeOH}$. The residual EtOAc fraction was subjected to silica gel column chromatography (CC) with a mixture of $n$-hexane-EtOAc to give 7 fractions (E1E7). Compound 2 (32 mg) was obtained from E1 by recrystallization using $\mathrm{MeOH}$. E2 was subjected to $\mathrm{CC}$ over silica gel eluted with a mixture of $\mathrm{CHCl}_{3}-\mathrm{MeOH}$ to give 9 subfractions (E21-E29). E27 was further subjected to CC over silica gel eluted with the mixture of $\mathrm{CHCl}_{3}-\mathrm{MeOH}$ to give 9 
subfractions (E271-E279). E277 was subjected to CC over Sephadex LH-20 eluted with $n$-hexane- $\mathrm{CH}_{2} \mathrm{Cl}_{2}-\mathrm{MeOH}$ ( $5: 5: 1)$ to give compound 3 (51 mg). Compound 4 (25 mg) was obtained from E3 by the CC over Sephadex LH-20 using $\mathrm{CH}_{2} \mathrm{Cl}_{2}-\mathrm{MeOH}(1: 1)$. E4 was subjected to $\mathrm{CC}$ over silica gel eluted with the mixture of $\mathrm{CHCl}_{3}-\mathrm{MeOH}$ to give 9 subfractions (E41-E49). E47 and E48 were subjected to $\mathrm{CC}$ over Sephadex LH-20 eluted with $\mathrm{CH}_{2} \mathrm{Cl}_{2}-\mathrm{MeOH}(1: 1)$ to give compounds 5 (19 mg) and 6 (24 mg).

Cell Culture and Differentiation Induction 3T3-L1 mouse embryo fibroblasts were obtained from the American Type Culture Collection (Manassas, VA, U.S.A.). Cells were cultured in Dulbecco's modified Eagle's medium (DMEM) supplemented with $10 \%$ fetal bovine serum (FBS) until confluence. Two days after confluence (day 0), cells were stimulated to differentiate with differentiation medium containing DMEM with 10\% FBS, 0.5 mM 3-isobutyl-1-methyl-xanthine, $1 \mu \mathrm{M}$ insulin and $1 \mu \mathrm{M}$ dexamethasone for $2 \mathrm{~d}$ (day 2). Cells were then maintained in DMEM supplemented with $10 \% \mathrm{FBS}$ and $1 \mu \mathrm{M}$ insulin for another $2 \mathrm{~d}$ (day 4), followed by culturing with DMEM with $10 \%$ FBS for an additional $4 \mathrm{~d}$ (day 8). All media contained $100 \mathrm{IU} / \mathrm{ml}$ penicillin and $100 \mu \mathrm{g} / \mathrm{ml}$ streptomycin. Cells were maintained at $37^{\circ} \mathrm{C}$ in a humidified atmosphere of $95 \%$ air $-5 \% \mathrm{CO}_{2}$.

The purity of each compound was verified $>95 \%$ by HPLC. Test compounds were dissolved in dimethyl sulfoxide (DMSO). Our preliminary study showed that DMSO at a final concentration of $0.1 \%$ in media did not affect cell viability or differentiation. The cultures were treated with test samples for whole culture period (day 0 -8) for general experiment. In some experiments, test samples were treated for indicated time-periods to evaluate their time dependent activity.

Oil Red O Staining Lipid droplets in cells were stained with Oil Red O. Eight days after differentiation induction, cells were washed three times with phosphate buffered saline (PBS) and fixed with 10\% formalin at room temperature for $1 \mathrm{~h}$. After fixation, cells were washed once with PBS and stained with freshly diluted Oil Red $\mathrm{O}$ solution (3 parts of $0.6 \%$ Oil Red O in isopropyl alcohol and 2 parts of water) for $15 \mathrm{~min}$. Cells were then washed twice with water and visualized. For quantitative analysis, Oil Red O staining was dissolved with isopropyl alcohol and optical density was measured at $520 \mathrm{~nm}$ by enzyme-linked immunosorbent assay (ELISA) plate reader.

Cell Viability Cell viability was assessed by 3-(4,5-dimethylthiazol-2-yl)-2.5-diphenyltetrazolium bromide (MTT) assay. 3T3-L1 cells were incubated with $0.5 \mathrm{mg} / \mathrm{ml}$ of MTT in the last $2 \mathrm{~h}$ of the culture period tested. Reduction of MTT to formazan was assessed by ELISA plate reader.

Western Blot Analysis Cells were lysed with sodium dodecyl sulfate (SDS) lysis buffer containing protease inhibitor cocktail. The lysates were centrifuged and supernatants collected. Proteins were separated in $10-15 \%$ SDSpolyacrylamide gel and transferred to a polyvinylidene difluoride (PVDF) membrane. After blocking in TBS-T with 5\% non-fat dry milk, the membrane was washed and incubated with primary antibodies including peroxisome proliferator activated receptor $\gamma(\operatorname{PPAR} \gamma)$ (Santa Cruz Biotechnology Inc., CA, U.S.A.) and $\beta$-actin at $4{ }^{\circ} \mathrm{C}$ overnight. After washing, the membrane was incubated with anti-rabbit horserad-
Table 1. Effects of Total Methanolic Extract and Each Fraction of $F$. rhynchophylla on Adipocyte Differentiation in 3T3-L1 Cells

\begin{tabular}{lc}
\hline \multicolumn{1}{c}{ Fraction } & Relative fat accumulation (\%) \\
\hline Undifferentiated control & $0.0 \pm 1.3$ \\
Differentiated control & $100.0 \pm 2.6$ \\
Total extract & $85.2 \pm 7.7$ \\
$n$-Hexane fraction & $88.1 \pm 6.2$ \\
$\mathrm{CHCl}_{3}$ fraction & $63.3 \pm 10.6^{*}$ \\
EtOAc fraction & $14.7 \pm 4.2^{* *}$ \\
$n$-BuOH fraction & $99.7 \pm 2.6$ \\
Aqueous fraction & $103.7 \pm 5.0$ \\
\hline
\end{tabular}

$* p<0.05 ; * * p<0.001$ compared with differentiated control.

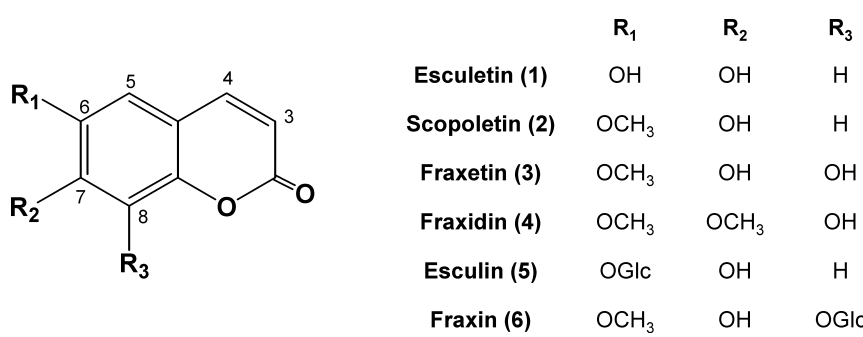

Fig. 1. Structures of Coumarins Isolated from F. rhynchophylla

ish peroxidase-conjugated immunoglobulin $\mathrm{G}$ ( $\mathrm{IgG}$ ) secondary antibody (Cell Signaling Technology Inc., MA, U.S.A.). The membrane was detected by chemiluminescent reaction, followed by exposure to X-ray Kodak film. PPAR $\gamma$ protein was normalized by $\beta$-actin and the intensities of bands were quantified by Scion-Image for Window Program.

Statistical Analysis Evaluation of statistical significance was determined by one-way ANOVA test with a value of $p<0.05$ considered statistically significant.

\section{RESULTS}

In the course of screening, the methanolic extract of $F$. rhynchophylla barks significantly inhibited the adipocyte differentiation. The methanolic extract was further fractionated into $n$-hexane, $\mathrm{CHCl}_{3}$, EtOAc and $n$-BuOH fractions. Among them, EtOAc fraction showed potent inhibitory activity on adipocyte differentiation at a concentration of $100 \mu \mathrm{g} / \mathrm{ml}$ (Table 1). Therefore, activity-guided fractionation of EtOAc was carried out for the isolation of active constituents. Further fractionation and separation of EtOAc fraction by several chromatographic methods yielded six coumarins, which were identified as esculetin (1), scopoletin (2), fraxetin (3), fraxidin (4), esculin (5) and fraxin (6) (Fig. 1) by direct comparison of their physicochemical and spectroscopic data with those previously reported. ${ }^{15,20-22)}$

The inhibitory activity of isolated compounds (1-6) on adipocyte differentiation was also evaluated in our assay system. Among the compounds isolated, esculetin (1) showed the most potent inhibitory activity, followed by fraxetin (3). At a concentration of $100 \mu \mathrm{M}$, esculetin (1) and fraxetin (3) inhibited adipocyte differentiation of $15 \%$ and $48 \%$ of fully differentiated cells, respectively (Fig. 2). Interestingly, both compounds showed little activity when treated to fully differentiated adipocytes (data not shown). However, these compounds did not show significant effects when treated to dif- 


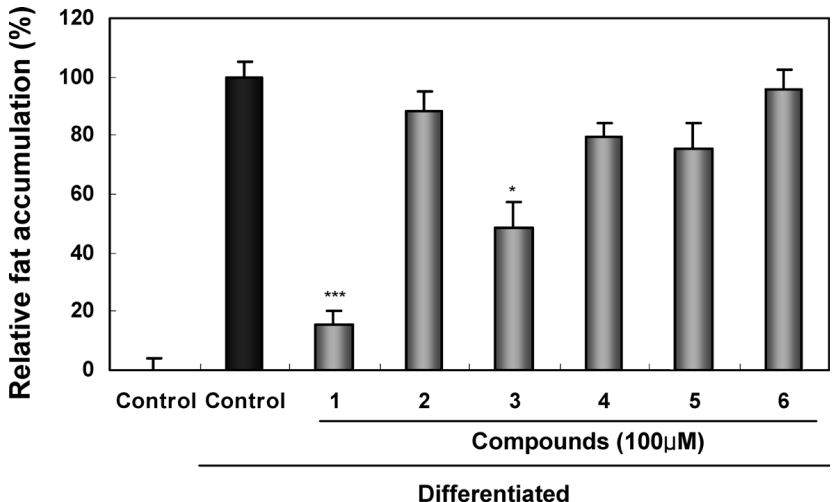

Fig. 2. Effects of Coumarins from F. rhynchophylla on Adipocyte Differentiation in 3T3-L1 Cells

Cultures were induced to differentiate for $8 \mathrm{~d}$. Cells were treated with coumarins for whole differentiation process (days $0-8$ ). On day 8 , cultures were subjected to Oil Red $\mathrm{O}$ staining. Percent adipocyte differentiation (\%) was calculated as $100 \times[$ (absorbance of sample-treated-absorbance of undifferentiated control)/(absorbance of differentiated control $\times$ absorbance of undifferentiated control)]. Results are expressed as mean \pm S.D. of three independent experiments, each performed using triplicate wells $* p<0.05 ; * * * p<0.001$ compared with differentiated control.

ferentiated adipocytes (data not shown). These results suggest that esculetin (1) and fraxetin (3) might be effective in the prevention of adipogenesis but not in lipolysis.

Adipogenic differentiation is a well organized process accompanied by dramatic alteration. This process occurs in several stages, which involves mitotic clonal expansion and a cascade of transcription factors. ${ }^{4,5,23)}$ Therefore, we further examined which stage of adipogenic differentiation was effectively inhibited by esculetin (1), the most active coumarin. During differentiation, 3T3-L1 cells were incubated with $100 \mu \mathrm{m}$ esculetin (1) at different periods of differentiation, namely, during days $0-2,2-4,4-8,0-4,2-8$ and $0-8$. On day 8 , cells were stained with Oil Red $\mathrm{O}$ and photographed. As shown in Fig. 3, esculetin (1) significantly inhibited adipocyte differentiation when treated during days $0-2,0-4$ and $0-8$. However, there was no significant inhibitory effect when treated during days $2-4,4-8$ and $2-$ 8. These results suggest that esculetin (1) could effectively inhibit the early stage of adipocyte differentiation.

Adipogenic differentiation involves expression of various transcriptional factors depending on differentiation stage. ${ }^{23)}$ Since esculetin (1) showed inhibitory effect on initial stage of adipocyte differentiation, we further investigated the effect of esculetin (1) on the early adipogenic transcription factor, PPAR $\gamma$. Esculetin (1) significantly blocked induction of $\operatorname{PPAR} \gamma$ expression in differentiated adipocytes, as measured by Western blot analysis (Fig. 4). We also checked the effect of esculetin (1) on PPAR $\gamma$ using troglitazone, a PPAR $\gamma$ agonist. Treatement of $5 \mu \mathrm{M}$ troglitazone significantly induced adipocyte differentiation assessed by fat accumulation. However, esculetin (1) at a concentration of $100 \mu \mathrm{M}$ significantly inhibited troglitazone-induced adipogenic differentiation (Fig. 5). These results suggest that esculetin (1) inhibited adipogenic differentiation, in part, via inhibition of PPAR $\gamma$-dependent pathway.

\section{DISCUSSION}

Obesity arises from abnormal growth of adipocyes leading
(A)

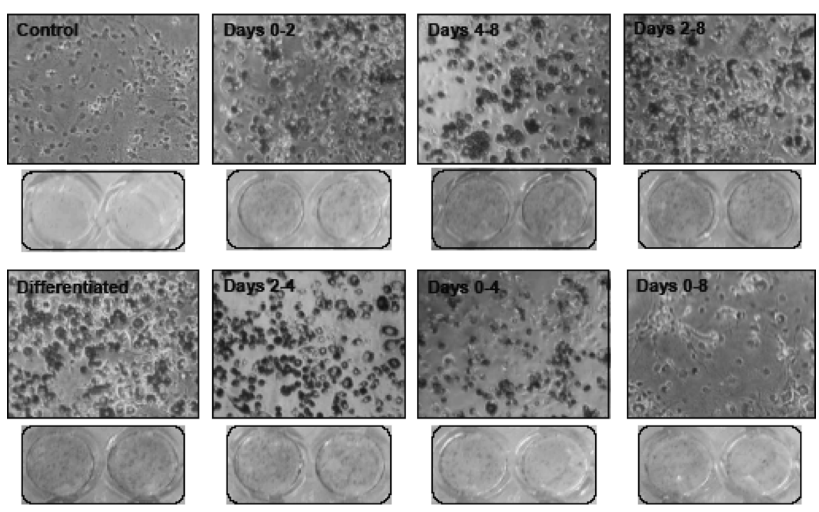

(B)

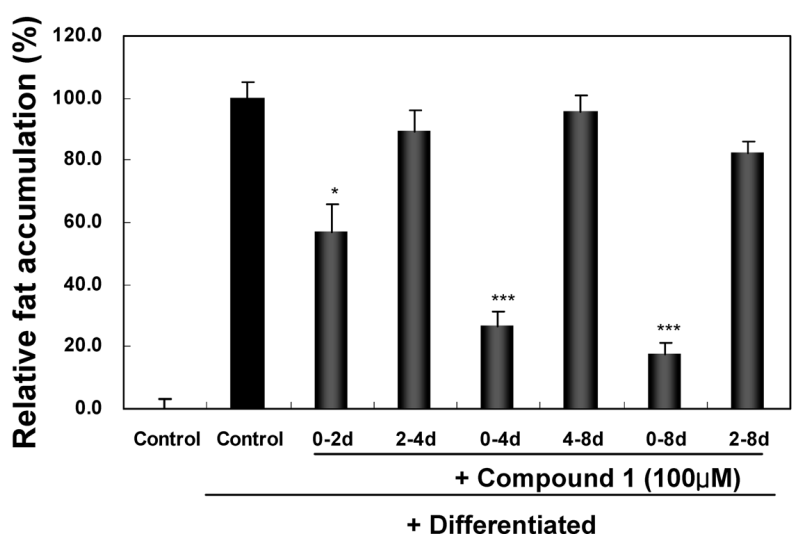

Fig. 3. Effects of Esculetin (1) on Adipocyte Differentiation during Differentiation Process

Cultures were induced to differentiate and esculetin $(100 \mu \mathrm{M})$ was added at different periods of differentiation, namely, during days $0-2,2-4,4-8,0-4,2-8$ and $0-8$. On day 8 , cells were stained with Oil Red O, photographed (A) and Oil Red O was quantitated (B). Figures are representatives of three independent experiments with similar results. Values are expressed as means \pm S.D. of triplicate experiments. $* p<0.05$; $* * * p<0.001$ compared with differentiated control.

to increased total fat mass. Abnormal growth of adipocytes, which is characterized by increased numbers of adipocytes containing lipids due to processes of mitogenesis and differentiation, is known associated with several pathological disorders including diabetes, hypertension, atherosclerosis and cancer. $^{1-3)}$

Adipocyte differentiation is an important process for cellular function in normal condition. However, pathological adipocyte differentiation confers abnormal expression of adipocytokines, which are implicated in insulin resistance and metabolic disorders. Thus, inhibition of adipocyte differentiation has been suggested an important therapeutic approach for prevention and treatment of obesity. Therefore, we searched for natural products having inhibitory activity on adipocyte differentiation. In the present study, we isolated six compounds from F. rhynchophylla and confirmed anti-adipogenic activity of two compounds from F. rhynchophylla, esculetin (1) and fraxetin (3), in 3T3-L1 cells.

Six compounds isolated from the stem barks of F. rhynchophylla have a simple coumarin skeleton and only differ in the substitutions in C-6, 7 and 8 positions (Fig. 1). Esculetin (1), which has two hydroxyl moieties at C-6, 7 positions, 


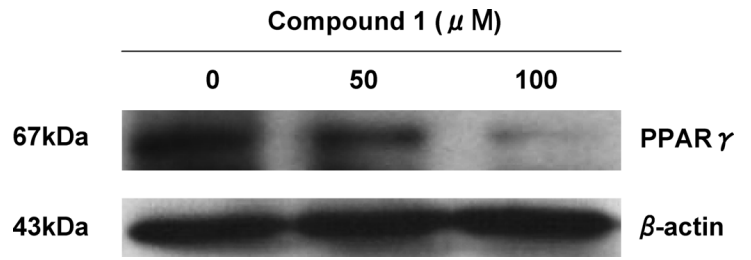

Fig. 4. Effect of Esculetin (1) on PPAR $\gamma$ Expression in 3T3-L1 Cells

Cultures were induced to differentiation with or without esculetin for $8 \mathrm{~d}$. On day 8 , PPAR $\gamma$ expression was measured by Western blot analysis. Data are representatives of three independent experiments.

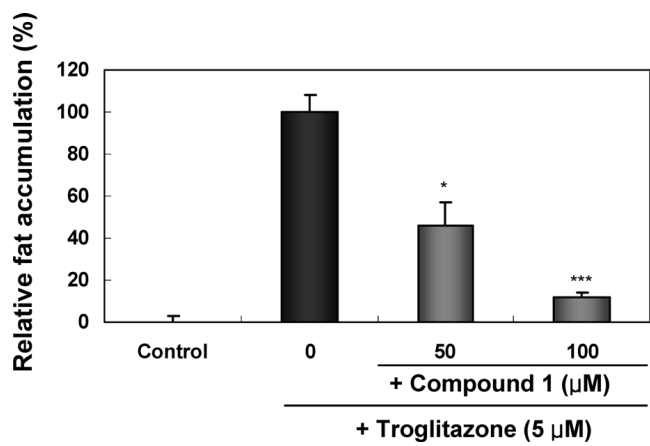

Fig. 5. Effect of Esculetin (1) on Troglitazone-Induced Adipocyte Differentiation

Cultures were induced to differentiation by treatment of $5 \mu \mathrm{M}$ troglitazone with or without esculetin $(100 \mu \mathrm{M})$ for $8 \mathrm{~d}$. On day 8 , cultures were subjected to Oil Red $O$ staining. The percent of adipocyte differentiation (\%) was calculated as $100 \times[(a b-$ sorbance of sample-treated-absorbance of undifferentiated control)/(absorbance of differentiated control-absorbance of undifferentiated control)]. Results are expressed as mean \pm S.D. of three independent experiments, each performed using triplicate wells $* * * p<0.001$ compared with differentiated control.

showed the most potent inhibitory activity in our assay system. Fraxetin (3), which has two hydroxyl moieties at C-7, 8 positions and a methoxyl moiety at C-6 position, also showed significant inhibitory activity. Interestingly, scopoletin (2), which has an identical structure to esculetin (1) except an additional methoxyl group at C-6 instead of hydroxyl moiety, showed weak activity. Moreover, addition of hydroxyl moiety to scopoletin (2) significantly increased the activity as shown in fraxetin (3), whereas addition of methoxyl moiety to fraxetin (3) significantly decreased the activity as shown in fraxidin (4). These results suggest that a free hydroxyl group of simple coumarin skeleton might be important for exerting anti-adipogenic activity. In addition, addition of glucose group to esculetin (1) and fraxetin (3), significantly decreased activity in our assay system as shown in esculin (5) and fraxin (6), respectively. Taken together, we cautiously suggest the importance of a hydroxyl group of coumarins related to anti-adipogenic acitivity, which should be clarified by assessing more derivatives.

Adipogenesis, the development of mature fat cells from preadipocytes, is an intensely studied model of cellular differentiation. The process of adipogenesis includes alteration of cell shape, growth arrest, clonal expansion, and a complex sequence of changes in gene expression and storage of lipid. ${ }^{20)}$ For differentiation, preadipocytes enter growth arrest and continue to subsequent differentiation process by appropriate mitogenic and adipogenic signals. During the initial stage of differentiation, a dramatic decrease of preadipocyte factor-1 (Pref-1) expression accompanies a rapid increase in the expression of PPAR $\gamma$ and CCAAT/enhancer-binding protein $(\mathrm{C} / \mathrm{EBP})$. During the terminal stage of differentiation, enzymes involved in triacylglycerol metabolism such as fatty acid synthase and glycerol-3-phosphate dehydrogenase increase to a great extent. ${ }^{4,53)}$ Therefore, we further examined which stage of adipogenic differentiation was effectively inhibited by esculetin (1), the most active coumarin. Interval treatment of esculetin (1) during differentiation induction showed that esculetin (1) exerted inhibitory activity on adipogenic differentiation when treated within $2 \mathrm{~d}$ (days $0-2$ ) after differentiation induction (Fig. 3). In addition, esculetin (1) was ineffective when treated to fully differentiated adipocytes. These results suggest that esculetin (1) might act on early stage of differentiation.

Since esculetin (1) showed inhibitory effect on initial stage of adipocyte differentiation, we further investigated the effect of esculetin (1) on PPAR $\gamma$, an early adipogenic transcription factor. PPAR $\gamma$ belongs to the nuclear receptor superfamily of ligand-activated transcriptional factors and is known necessary and sufficient for adipocyte differentiation. ${ }^{24,25)}$ Esculetin (1) significantly blocked induction of PPAR $\gamma$ expression in differentiated adipocytes, as measured by Western blot analysis (Fig. 4). In addition, esculetin (1) significantly inhibited adipocyte differentiation induced by troglitazone, a PPAR $\gamma$ agonist (Fig. 5). These results suggest that esculetin (1) inhibited adipogenic differentiation, in part, via inhibition of PPAR $\gamma$-dependent pathway.

In conclusion, the present study suggests that EtOAc fraction and coumarins isolated from $F$. rhynchophylla inhibit adipocyte differentiation in 3T3-L1 cells by decreasing fat accumulation. Thus, it will be of interest further to test whether these coumarins decrease adipocyte differentiation in vivo, for example, in animal models of obesity, to explore their therapeutic potential for obesity. This will provide further insight into the design of new approaches to obesity.

Acknowledgement This research was supported by the Korea Research Foundation (KRF) Grant funded by the Korea government (MEST) (No. 2009-0068688) and the Grant of the Korean Ministry of Education, Science and Technology [The Regional Core Research Program/Chungbuk BIT Research-Oriented University Consortium].

\section{REFERENCES}

1) Kopelman P. G., Nature (London), 404, B635-B643 (2000).

2) Visscher T. L., Seidell J. C., Annu. Rev. Public Health, 22, 355-375 (2001).

3) Kruger J., Galuska D. A., Serdula M. K., Jones D. A., Am. J. Prevent. Med., 26, 402-406 (2004).

4) Rosen E. D., Spiegelman B. M., Nature (London), 444, 847-853 (2006).

5) Farmer S. R., Genes Dev., 22, 1269-1275 (2008).

6) Trayhurn P., Acta Physiol. Scand., 184, 295-293 (2008).

7) Rayalam S., Della-Fera M. A., Baile C. A., J. Nutr. Biochem., 19, $717-726$ (2008).

8) Moon H.-S., Chung C.-S., Lee H.-G., Kim T.-G., Choi Y.-J., Cho C.S., Obesity, 15, 2571-2582 (2007).

9) Ahn J., Lee H., Kim S., Park J., Ha T., Biochem. Biophys. Res. Commun., 373, 545-549 (2008).

10) Rayalam S., Yang J.-Y., Ambati S., Della-Fera M. A., Baile C. A., Phytother. Res., 22, 1367-1371 (2008).

11) Lee M.-S., Kim C.-T., Kim I.-H., Kim Y., Phytother. Res., 28, 1088 1091 (2009). 
12) Kim N. Y., Pae H. O., Ko Y. S., Yoo J. C., Choi B. M., Jun C. D., Chung H. T., Inagaki M., Kiguchi R., Kim Y. C., Planta Med., 65, 656-658 (1999)

13) Bae K. H., "The Medicinal Plants of Korea," Kyo-Hak Publishing, Seoul, 1999.

14) Wright C. I., Van-Buren L., Kroner C. I., Koning M. M., J. Ethnopharmacol., 114, 1-31 (2007).

15) Thuong P. T., Pokharel Y. R., Lee M. Y., Kim S. K., Bae K. H., Su N. D., Oh W. K., Kang K. W., Biol. Pharm. Bull., 32, 1527-1532 (2009).

16) Jiang J. H., Jin C. M., Kim Y. C., Kim H. S., Park W. C., Park H., Biol. Pharm. Bull., 31, 2273-2276 (2008).

17) Kim N. Y., Ho P., Ko Y. S., Yoo J. C., Choi B. M., Jun C. D., Chung H. T., Inagaki M., Higuchi R., Kim Y. C., Planta Med., 65, 656-658 (1999).

18) Kostova I., Lossifova T., Fitoterapia, 78, 85-106 (2007).
19) Lin S., Wang S., Liu M., Gan M., Li S., Yang Y., He W., Shi J., J. Nat. Prod., 70, 817-823 (2007).

20) Yasuda T., Fukui M., Nakazawa T., Hoshidawa A., Ohsawa K., J. Nat. Prod., 69, 755-757 (2006).

21) Kim J. S., Kim J. C., Shim S. H., Lee E. J., Jin W., Bae K. H., Son K. H., Kim H. P., Kang S. S., Chang H. W., Arch. Pharm. Res., 29, 617623 (2006).

22) Thuong P. T., Hung T. M., Ngoc T. M., Ha D. T., Min B. S., Kwack S. J., Kang T. S., Choi J. S., Bae K. H., Phytother. Res., 24, 101-106 (2010).

23) Lefterova M. I., Lazar M. A., Trends Endocrinol. Metab., 20, 107114 (2009).

24) Lehrke M., Lazar M. A., Cell, 123, 993 -999 (2005).

25) Tontonoz P., Spiegelman B. M., Annu. Rev. Biochem., 77, 289-312 (2008). 